\title{
Sex Differences in Heritability of Neck Pain
}

\author{
René Fejer, ${ }^{1,2}$ Jan Hartvigsen, ${ }^{1,3}$ and Kirsten Ohm Kyvik ${ }^{2}$ \\ I Institute of Sports Science and Clinical Biomechanics, University of Southern Denmark, Odense, Denmark \\ ${ }^{2}$ Institute of Public Health, Epidemiology, The Danish Twin Registry, University of Southern Denmark, Odense, Denmark \\ ${ }^{3}$ Nordic Institute of Chiropractic and Clinical Biomechanics, Odense, Denmark
}

$E^{x}$ xperimental studies have suggested biological factors as a possible explanation for gender disparities in perception of pain. Recently, heritability of liability to neck pain (NP) has been found to be statistically significantly larger in women compared to men. However, no studies have been conducted to determine whether the sex differences in heritability of NP are due to sex-specific genetic factors. Data on lifetime prevalence of NP from a populationbased cross-sectional survey of 33,794 Danish twins were collected and age-stratified univariate biometrical modeling using sex-limitation models was performed based on 10,605 dizygotic (DZ) twins of opposite sex to estimate the qualitative sex differences. In a full sex-limitation model the genetic component in females were higher than in males, but the genetic and the shared environmental correlations were equal to what is normally assumed between same-sex DZ twins. A 'no-sex-effects' model showed the overall best model fit which confirms absence of sex-related gene interaction. The age-stratified sex-limitation models showed similar results. Thus, there is no evidence for a sex-specific genetic influence in the liability of heritability of NP.

Neck pain (NP) is more common in women than men (Ariëns et al., 1999; Côté et al., 1998; Fejer, Kyvik, et al., in press). In a systematic critical literature review a higher prevalence for women was reported in $83 \%$ of all included studies (Fejer, Kyvik, et al., in press). In addition, women with NP have more days and longer duration of sick leave than men (Leijon et al., 1998). Several physical and psychosocial factors in relation to work conditions have been suggested to be explanations for these gender disparities (Bingefors \& Isacson, 2004; Fredriksson et al., 1999; Leijon et al., 1998). Furthermore, experimental studies on mechanical pressure pain thresholds have shown that females have a lower pain threshold than men and it is suggested that this reduced pain threshold leads to increased risk of musculoskeletal pain (Riley et al., 1998; Rollman \& Lautenbacher, 2001). The increased pain sensitivity and decreased pain tolerance in women points to biological factors as a possible explanation for the gender disparities (Riley et al., 1998; Rollman et al., 2004).
Research in the genetics of pain is steadily growing and of special interest is identifying pain susceptibility genes for complex conditions like NP (Mogil \& Devor, 2004). A recent study on the heritability of lifetime NP in a large Danish twin cohort study (the Omnibus 2002 twin cohort survey — for details see below) showed that the genetic contribution was statistically significantly larger in women $(52 \%, 95 \%$ confidence intervals [CI] $47-58)$ vs. men $\left(34 \%, 95 \%\right.$ CI $26-41 ; \chi^{2}=174.1, p<.0001$; Fejer, Hartvigsen, et al., in press). The genetic component became gradually less important with increasing age and for females the environmental influences dominated completely in the older age groups. Unfortunately, these heritability estimates only refer to the extent to which genetic (and environmental factors) in the population account for the phenotypic differences (i.e., having NP or not). In other words, these quantitative measures do not reveal if men and women are influenced by the same genetic and environmental factors or whether they are influenced by different genes or different environmental exposures.

A unique way to examine whether men and women are influenced by different genetic and environmental factors is by including dizygotic twin pairs of opposite sex (DZos) in the analysis. It is assumed that samesexed dizygotic twin pairs (DZss) share the same environment and half their genes like normal siblings (e.g., the shared environmental correlation is 1.00 and the genetic correlation .5). If NP in DZos twins correlates less than for NP in DZss twins then this difference is possibly due to gender. Thus, the genetic correlation between the sexes refers to the extent to which the same genetic factors affect men and women regardless of the heritability estimates in general.

The objective of this article is to examine whether the gender difference in heritability of NP is due to sex-specific different genetic factors. This article will add to further understanding of how complex traits such as NP may be influenced by genetic factors.

Received 2 June, 2005; accepted 6 December, 2005.

Address for correspondence: René Fejer, Institute of Sports Science and Clinical Biomechanics, University of Southern Denmark, Campusvej 55, DK-5230 Odense M, Denmark.E-mail: rfejer@health.sdu.dk 


\section{Materials and Methods \\ Study Sample}

In April 2002 a questionnaire was sent out to 46,418 twins between the age of 20 and 71 years followed by a reminder approximately 3 weeks later. All the twins were registered in the Danish twin Registry (DTR) and had prior to this study given their consent to participate in research projects. The twins in the DTR are considered representative of the general population in Denmark in terms of many diseases and mortality (Christensen et al., 1995; Kyvik, 2000; Skytthe et al., 2002). The zygosity of the twins in this cohort has previously been determined via self-reported questionnaires (Christiansen et al., 2003). The accuracy of determining a correct zygosity classification within this cohort was $97 \%$ which is considered acceptable.

In total, 35,315 (76\%) returned the questionnaire, but only 33,794 questionnaires were eligible for further analysis due to blanks, doublets, ID number removal, and so forth.

\section{Measures}

The lifetime prevalence was used in order to avoid possible short-term time-specific NP ('Have your ever had neck trouble?'; Kuorinka et al., 1987). The definition of NP ('ache, pain, or discomfort') was stated in the questionnaire and supplemented by a drawing showing the anatomical area of the neck (between occiput and the third thoracic vertebra, and between the scapulae).

\section{Statistical Analyses}

Twins were classified into five categories according to gender and zygosity: monozygotic male twins (MZM), monozygotic female twins (MZF), dizygotic male twins (DZM), dizygotic female twins (DZF), and DZos. In total, 30,341 twins had answered the question on lifetime NP prevalence (MZM: 3596; MZF: 4618; DZM: 5395; DZF: 6127; DZos: 10,605 twins).

The phenotypic analyses of gender-specific MZ and DZ twins (e.g., prevalence, probandwise concordance rates, and tetrachoric correlations) have previously been presented by Fejer, Hartvigsen, et al. (in press) and only results relevant in this paper are introduced together with results from DZos. The NP prevalence estimates showed a tendency to increase until the age of about 35 where no age effect was seen until the age of about 52 where a decrease in prevalence reporting was noted. A linear logistic regression analysis would therefore not seem appropriate and thus, all analyses were performed using age stratifications (20-35 years, 36-50 years, and 51-71 years).

A series of univariate structural equation modeling analyses were fitted to test whether the relative importance of genetic and environmental influences differs between males and females (Neale \& Cardon, 1992). In general, any resemblance for a measured trait (NP) between same-sex twins can be due to genetic factors (either additive - A, or dominant - D), shared/common environmental factors (C), and any nonshared environmental factors (E) that make each twin unique. However, a model in which all parameters are incorporated (i.e., an ACDE model) is not possible due to the confounding between A, C, and D components. Therefore, usually two separate models (the ACE and ADE models) are estimated and the best fit (see below) is chosen. In Fejer, Hartvigsen, et al.'s (in press) study the ACE model showed the best fit (varying between $\mathrm{AE}$ and $\mathrm{CE}$ according to different age groups), and thus is the only model considered in this paper. An ACE univariate model, composed of three population-based variance components needs therefore to be calculated for each same-sexed twin category (see above) using structural equation modeling (Neale et al., 1992). The sex-limitation models are similar to univariate models but are designed to also include DZos. By including DZos the genetic and the shared environmental correlations for DZos can be compared with DZss. If the DZos correlations are smaller than for DZss this indicates existence of factors that contribute to individuals differences in one sex, but not the other sex, hence a qualitative sex difference is estimated.

Two different sex-limitation models were used: the general (full) model and the common effects model. In the general sex-limitation model the genetic or the environmental correlation were freely estimated (Figure 1a). In other words, the magnitude of the common effects need not be the same for both the males and the females and thus, the sex-specific additive genetic and the shared environmental influences are estimated. In addition, the female-male genetic correlation $\left(r_{\mathrm{G}}\right)$ was calculated in order to determine the extent of female-male genetic similarity. In the common effects model both correlations are fixed (i.e., the environmental correlation fixed to 1.00 and the genetic correlation fixed to .5; Figure 1b). If the DZos covariance for the common effects model is statistically significantly less compared to the full model then there is evidence for sex-specific effects. Finally, in the no-sex-difference model each variance component is equaled between male and females (i.e., $\mathrm{a}^{2} m=\mathrm{a}^{2} f, \mathrm{c}^{2} m=\mathrm{c}^{2} f, \mathrm{e}^{2} m=\mathrm{e}^{2} f$ ) and both correlations are fixed, as in the common effects model $\left(r_{\mathrm{G}}=.5, r_{\mathrm{C}}=1.00\right)$. This is not an actual sex-limitation model but tests if a no-sex-difference model has better fit than the sex-limitation models. All nested sex-limitation models were compared to the full sex-limitation model, and for each sex-limitation model parsimonious (nested) models (i.e., AE and CE models) were tested using likelihood-ratio $\chi^{2}$ difference test $(\Delta-2 \mathrm{LL})$ with degrees of freedom equal to the differences in parameters and using the Akaike's Information Criteria (AIC; Akaike, 1974). Lower values represent better fitting models. All biometrical modeling analyses were further stratified into three age groups based on the variation in lifetime NP prevalence (Fejer, Hartvigsen, et al., in press) and were performed on raw data using the $\mathrm{Mx}$ (version 1.57a) software package (Neale et al., 2002). 
(a)

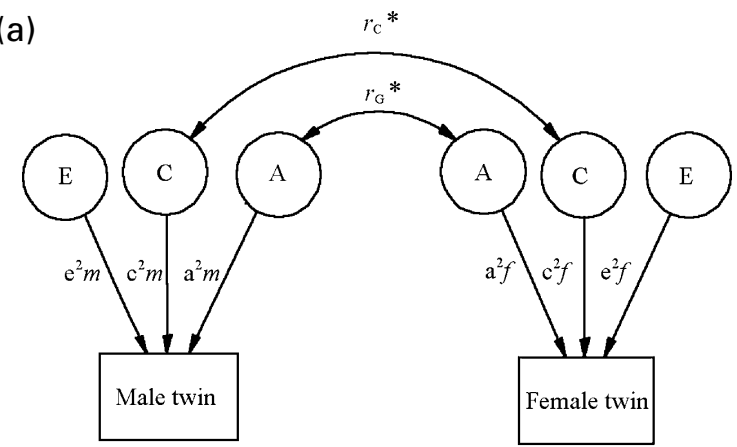

(b)

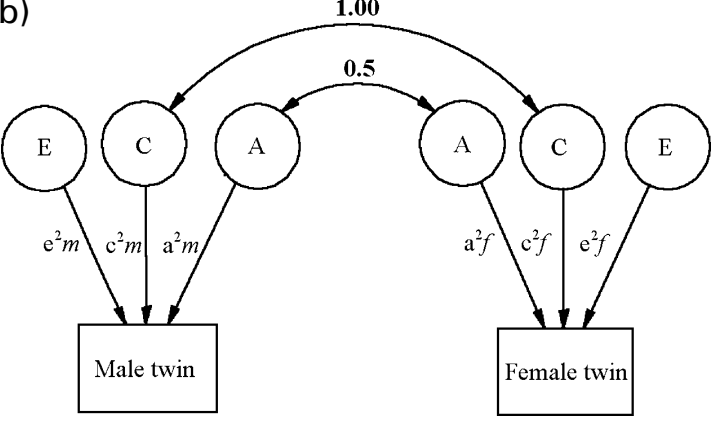

Figure 1

Path diagrams sex-limitation models illustrating parameters estimated in opposite-sex twin pairs.

Note: Rectangles enclose the observed (measured) variables. Circles show the latent (unmeasured) variables: additive genetic factor (A), shared environmental factor (C), and nonshared environmental factor (E). Single-headed arrows define causal relations between latent and observed variables. Double-headed arrows represent correlations between variables. $\mathrm{a}^{2} m, \mathrm{c}^{2} m, \mathrm{e}^{2} m=$ additive genetic, shared, and nonshared environmental estimates for males respectively; $\mathrm{a}^{2} f, \mathrm{c}^{2} f$, $\mathrm{e}^{2} f=$ additive genetic, shared, and nonshared environmental estimates for females respectively; $r_{\mathrm{G}}=$ genetic correlation; $r_{\mathrm{c}}=$ shared environmental correlation.

${ }^{*}$ Only one correlation is estimated at a time while the other correlation is fixed $\left(r_{\mathrm{G}}=.5, r_{\mathrm{C}}=1.00\right)$.

\section{Results}

In total $44.4 \%$ reported having had NP during their lifetime $(95 \%$ CI $43.8-44.9)$. The prevalence and tetrachoric correlations for each of the five twin categories are shown in Table 1 . The NP prevalence for women was statistically significantly higher compared to men (Fisher's exact test, $p<.001$ ) and MZ correlations were more than twice the correlation of DZ twins indicating a general genetic contribution. In addition, the female MZ and DZ correlations were higher than male correlations which suggest a possible sex-specific genetic influence. On the other hand, the higher correlation between DZos (male-female) compared to same-sexed DZ twins does not support a sex-specific influence in the heritability of NP.

In Table $2 \mathrm{a}$, biometric model fitting for each sexlimitation model is presented. In the full sex-limitation models (models 1 and 2) for all twins, the additive genetic component in females $\left(\mathrm{a}^{2} f\right)$ was higher than in males $\left(\mathrm{a}^{2} m\right)$, but the genetic $\left(r_{\mathrm{G}}\right)$ and the shared environmental correlations $\left(r_{\mathrm{C}}\right)$ were equal to what is normally assumed between same-sex DZ twins $\left(r_{\mathrm{G}}=.50\right.$

\section{Table 1}

Lifetime Neck Pain Prevalence and Tetrachoric Correlations for Five Zygosity Categories

\begin{tabular}{lccc}
\hline Zygosity group & $N$ & $\begin{array}{c}\text { Prevalence of NP } \\
\%(95 \% \mathrm{CI})\end{array}$ & $\begin{array}{c}\text { Tetrachoric correlation } \\
(95 \% \mathrm{Cl})\end{array}$ \\
\hline MZ males & 3596 & $35.3(33.7-36.8)$ & $0.41(0.33-0.49)$ \\
DZ males & 5395 & $36.1(34.8-37.3)$ & $0.16(0.07-0.24)$ \\
MZ females & 4618 & $49.1(47.6-50.5)$ & $0.49(0.42-0.55)$ \\
DZ females & 6127 & $51.8(50.6-53.1)$ & $0.23(0.17-0.30)$ \\
DZosmf & 10,605 & $45.3(44.4-46.3)$ & $0.33(0.28-0.39)$ \\
\hline
\end{tabular}

Note: $N=$ number of twin individuals who answered the NP questions; $\mathrm{MZ}=$ monozygotic twins; $\mathrm{DZ}=$ dizygotic twins; $\mathrm{DZosmf}=$ dizygotic twins with opposite sex (first twin is male and second twin is female). and $\left.r_{\mathrm{C}}=1.00\right)$. The 'no-sex-effects' model showed the overall best model fit $(\Delta-2 \mathrm{LL}=4.81, \Delta d f=4$, AIC -3.19), which confirms absence of sex-related gene interaction. The age-stratified sex-limitation models showed similar results (Tables $2 \mathrm{~b}-2 \mathrm{~d}$ ). Thus, this analysis does not support a sex-specific genetic influence in the liability of heritability of NP.

\section{$\overline{\text { Discussion }}$}

When investigating the heterogeneity between genders two questions arise: (1) is the contribution of genetic/environmental factors greater or smaller in males than females? And (2) are there different genetic/environmental factors influencing the trait in males and females? The first question is a quantitative measure (i.e., a heritability estimate) and was examined in the paper by Fejer, Hartvigsen, et al.'s (in press). The heritability of NP in females was indeed found to be statistically significantly higher than in males. The second question is a qualitative measure and was examined in the present article by also including DZos twin pairs in the analyses. It was demonstrated that the gender differences in heritability of NP was not due to any sex-specific genetic influence.

But if there is biological evidence for gender difference in pain perception why was this not seen in the present study? NP is a complex condition with a great psychological and social association, which has been shown to influence the genetic liability to NP (MacGregor et al., 2004). Early pain occurrence in women may result in sensitization both neurally and behaviorally according to the biological plasticity model suggested by Rollman et al. (2004). Accordingly, the sex-specific difference can be described as a genetic/behavioral difference rather than a pure sex-specific genetic difference in relation to pain. 


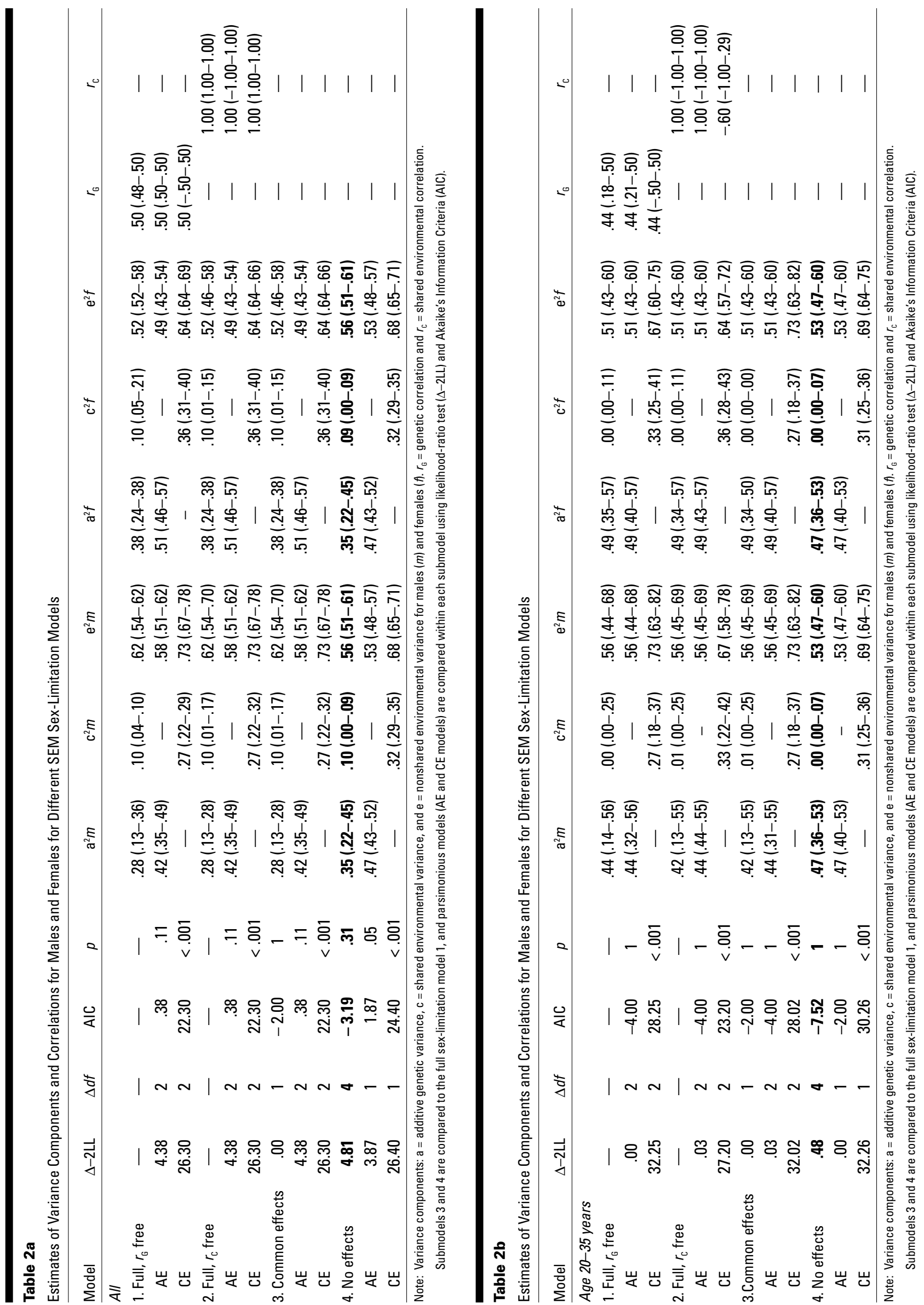




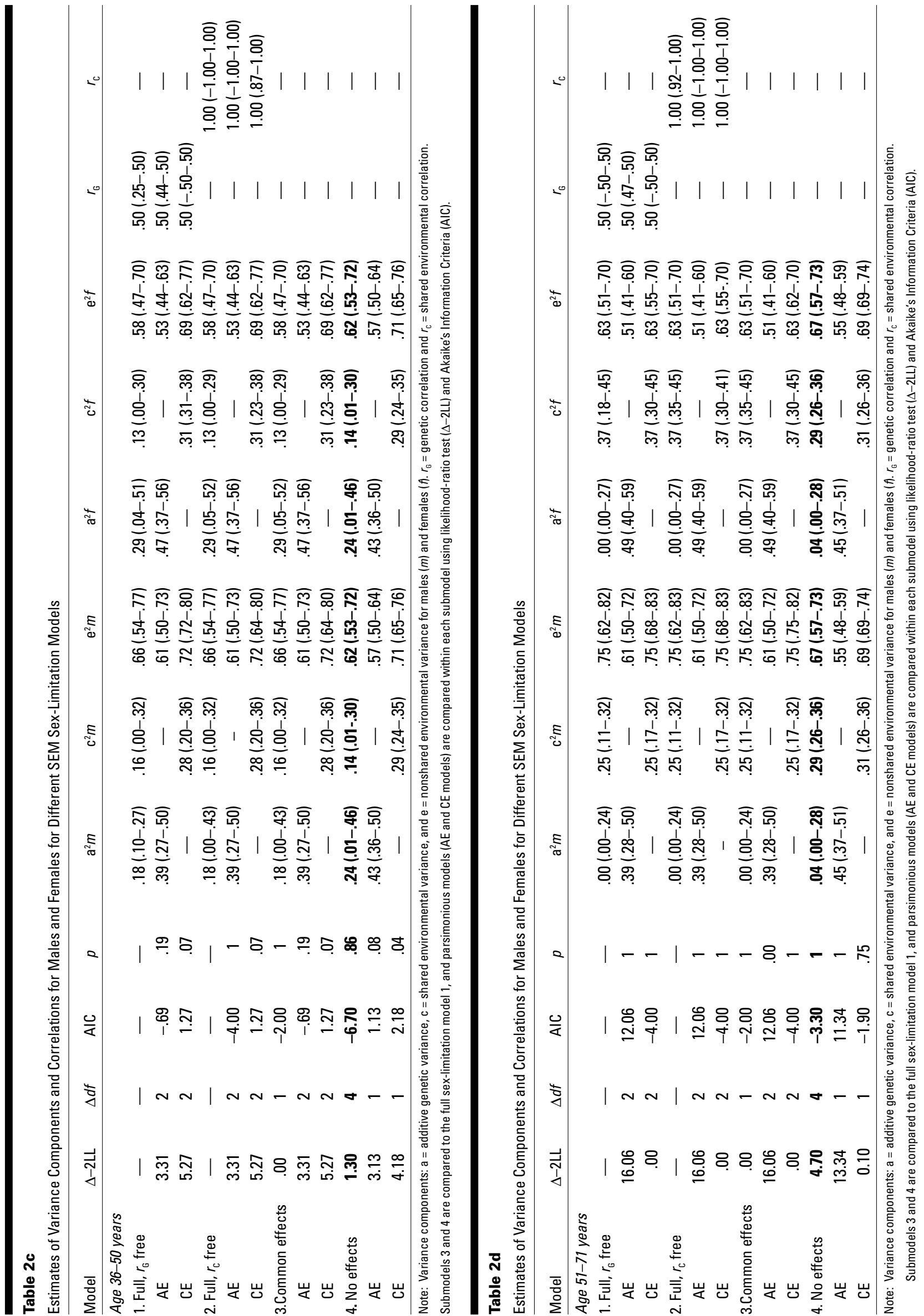


Therefore, the use of self-reported lifetime NP prevalence as a single phenotypic definition may not show possible influence of sex-related genetic components. Furthermore, the self-reported lifetime NP covers a wide range of more or less well-defined conditions and may thus be considered a heterogeneous cluster of etiologic pathologies and psychosocial conditions. A more clearly (clinically) defined phenotype of NP may possibly show a sex-specific genetic influence, and future studies within this field are therefore warranted in order to come closer to the understanding of how genetic factors are related to NP.

There are potential limitations to this study that need to be addressed. First, the phenotypic definition (e.g., self-reported lifetime NP prevalence) cannot be verified and may thus be considered unspecific and subject to recall bias. This means that although pain and the anatomical area of interest were defined, the cause or any underlying pathologic conditions are not known. However, the lack of clinically well-defined cases of NP and potential recall bias are assumed to be the same for MZ, DZ, and DZos twins and therefore not of concern in this study in terms of our structural equation modeling analyses. On the other hand, the existence of a specific and genetically determined underlying pathologic condition that may be possible, however, no such condition has yet been found and specific subgroups cannot be isolated and further analyzed in our study. Second, the self-reporting of lifetime NP may be influenced by recall bias. This potential recall bias may partly explain the decline in NP prevalence with increasing age. On the other hand, this may also be explained by a birth cohort due to different exposures at different points in time. For example, the elderly people may not have been significantly exposed to the same kind of occupational risk factors that have been associated with the development of NP today (Ariens et al., 2000). However, as this age-related decline in NP prevalence was seen in both genders, it does not affect the results derived from the biometric analyses.

Despite these limitations, this study has several strengths. The sex-specific analyses were based on a large population-based sample of twins aged 20 to 71 years. Therefore, results can be interpreted with confidence and subsequently extrapolated to the background population for a wide age range.

Future studies on genetic influences on NP should have two major focuses: (1) to examine the extent of heritability within subdefinitions of NP in different populations, and (2) include additional information such as, for example, psychosocial factors that are relevant for the basis of genetics of NP (i.e., anxiety and depression).

\section{Conclusion}

The higher prevalence and the significantly larger heritability of NP in women are not related to sex-specific genetic differences.

\section{Acknowledgments}

This study was supported by the Foundation of Chiropractic Research and Post Graduate Education and the European Chiropractic Union Research Fund. The authors wish to thank Inge Petersen, MSc, the Danish Twin Registry, and Jacob Hjelmborg, MSc, PhD, Department of Statistics, for assistance with the analyses.

\section{References}

Akaike, H. (1974). A new look at the statistical model identification. IEEE Transactions on Automatic Control, 19, 719-723.

Ariëns, G. A. M., Borghouts, J. A. J., \& Koes, B. W. (1999). Neck pain. In I. K. Crombie, P. R. Croft, S. J. Linton, L. LeResche, \& M. von Korff (Eds.), Epidemiology of pain (pp. 235-256). Seattle: IASP Press.

Ariens, G. A., van Mechelen, W., Bongers, P. M., Bouter, L. M., \& van der Wal, G. (2000). Physical risk factors for neck pain. Scandinavian Journal of Work, Environment and Health, 26, 7-19.

Bingefors, K., \& Isacson, D. (2004). Epidemiology, comorbidity, and impact on health-related quality of life of self-reported headache and musculoskeletal pain: A gender perspective. European Journal of Pain, 8, $435-450$.

Christensen, K., Vaupel, J. W., Holm, N. V., \& Yashin, A. I. (1995). Mortality among twins after age 6: Fetal origins hypothesis versus twin method. British Medical Journal, 310, 432-436.

Christiansen, L., Frederiksen, H., Schousboe, K., Skytthe, A., von Wurmb-Schwark, N., Christensen, K., \& Kyvik, K. (2003). Age- and sex-differences in the validity of questionnaire-based zygosity in twins. Twin Research, 6, 275-278.

Côté, P., Cassidy, J. D., \& Carroll, L. (1998). The Saskatchewan Health and Back Pain Survey. The prevalence of neck pain and related disability in Saskatchewan adults. Spine, 23, 1689-1698.

Fejer, R., Hartvigsen, H., \& Kyvik, K. O. (in press). Heritability of neck pain: A population-based study of 33,794 Danish twins. Rheumatology (published online).

Fejer, R., Kyvik, K. O., \& Hartvigsen, H. (in press). The prevalence of neck pain in the world population: A systematic critical review of the literature. European Spine Journal (published online).

Fredriksson, K., Alfredsson, L., Koster, M., Thorbjornsson, C. B., Toomingas, A., Torgen, M., \& Kilbom, A. (1999). Risk factors for neck and upper limb disorders: Results from 24 years of follow up. Occupational and Environmental Medicine, 56, 59-66.

Kuorinka, I., Jonsson, B., Kilbom, A., Vinterberg, H., Sorensen, F. B., Andersson, G., \& Jorgensen, K. (1987). Standardised Nordic Questionnaires for the analysis of musculoskeletal symptoms. Applied Ergonomics, 18, 233-237. 
Kyvik, K. O. (2000). Generalisability and assumptions of twin studies. In T. D. Spector, H. Snieder, \& A. J. MacGregor (Eds.), Advances in twin and sib-pair analysis (1st ed., pp. 67-87). London: Greenwich Medical Media.

Leijon, M., Hensing, G., \& Alexanderson, K. (1998). Gender trends in sick-listing with musculoskeletal symptoms in a Swedish county during a period of rapid increase in sickness absence. Scandinavian Journal of Social Medicine, 26, 204-213.

MacGregor, A. J., Andrew, T., Sambrook, P. N., \& Spector, T. D. (2004). Structural, psychological, and genetic influences on low back and neck pain: A study of adult female twins. Arthritis and Rheumatism, 51, 160-167.

Mogil, J. S., \& Devor, M. (2004). Introduction to pain genetics. In J. S. Mogil (Ed.), The Genetics of pain (pp. 1-17). Seattle: IASP Press.

Neale, M. C., Boker, S. M., Xie, G., \& Maes, H. (2002). Mx: Statistical modeling (6th ed.). Richmond, VA:
Virgina Commonwealth University, Department of Psychiatry.

Neale, M. C., \& Cardon, L. R. (1992). Methodology for genetic studies of twins and families. Dordrecht: Kluwer Academic Publisher.

Riley, J. L., Robinson, M. E., Wise, E. A., Myers, C. D., \& Fillingim, R. B. (1998). Sex differences in the perception of noxious experimental stimuli: A meta-analysis. Pain, 74, 181-187.

Rollman, G. B., Abdel-Shaheed, J., Gillespie, J. M., \& Jones, K. S. (2004). Does past pain influence current pain: Biological and psychosocial models of sex differences. European Journal of Pain, 8, 427-433.

Rollman, G. B., \& Lautenbacher, S. (2001). Sex differences in musculoskeletal pain. The Clinical Journal of Pain, 17, 20-24.

Skytthe, A., Kyvik, K., Holm, N. V., Vaupel, J. W., \& Christensen, K. (2002). The Danish Twin Registry: 127 birth cohorts of twins. Twin Research, 5, 352-357. 